\title{
Epidemiological study of genetic diversity and patterns of gene flow in Haemonchus species affecting domestic ruminants in Egypt
}

\author{
Omnia M. Kandil ${ }^{1 *}$, Khaled A. Abdelrahman ${ }^{1}$, Nagwa A. Eid ${ }^{2}$, Lobna M. Elakabawy ${ }^{2}$ and Mohamed A. Helal ${ }^{1}$
}

\begin{abstract}
Background: Haemonchosis is a major parasitic disease in Egyptian sheep industry and its effect on production, animal wellbeing, and welfare is likely to increase. The present study recorded Haemonchus spp. hereditary diversity and population structure among various animal hosts by amplification and sequencing of mitochondrial DNA(mtDNA) cytochrome oxidase subunit I (COI) gene distinguished at 709 base pair (bp) which have been submitted in GenBank with accession numbers (KT826575, KT826574, KT826573, and KT826572) for sheep, goats, cattle, and camels, individually.

Results: The main identity percent was $93.5 \%$ among sheep and goat isolates with divergence percent of $4.4 \%$. The most reduced identity percent was $80.2 \%$ among sheep and camel isolates with divergence percent of $21.9 \%$. The phylogenetic tree indicated clustering of sheep, goats, and cattle isolates which proved that high rates of gene flow among population and in between various ruminant hosts are existing as a result of intensively managed flocks. In contrast, Haemonchus longistipes (H. longistipes) confined from Egyptian camels indicated little homology with Haemonchus contortus (H. contortus) and was the hereditarily most distinct taxa without clustering with different hosts in phylogenetic analysis. The COI haplotypes from Egypt that were contrasted with Haemonchus isolates from different countries to elucidate the population structure revealed that our isolates indicated most elevated identity with Haemonchus isolated from Pakistan.

Conclusions: These results can be figured out as a part of a new control approach for haemonchosis incorporating and respecting ecological trends. This work is the principal focus at the molecular level which demonstrated that $H$. longistipes is Haemonchus spp. of Egyptian camels.
\end{abstract}

Keywords: Haemonchus, Genetic diversity, Gene flow, COl, Ruminants, H. Longistipes, Egypt

\section{Background}

Small ruminant production plays pivotal role in the domesticated animals industry around the world (Whitley et al. 2014). Sheep can be parasitized by a different scope of parasites, with well in excess of 150 internal and external species reported worldwide (Taylor 2010). In Egypt, Haemonchus contortus infection in sheep was recorded with high incidence (Elshahawy et al. 2014; Kandil et al. 2015). Haemonchus parasites cause high mortality rates and

\footnotetext{
* Correspondence: kandil_om@yahoo.com

1 Department of Parasitology and Animal Diseases, National Research Centre (NRC), PO Box 12622, 33 Bohouth St., Dokki, Giza, Egypt

Full list of author information is available at the end of the article
}

extraordinary efficiency diminishment rather than progressive elevations in curative costs. It has likewise been demonstrating great resistance against most of the anthelmintics presently being used (Schafer et al. 2015).

Genetic characterization and adoption of genome sequencing procedures are essential procedures for accurate identification (Gasser et al. 2008). The factors of hereditary structure of Haemonchus populations include geographical location, efficient population extents, host movement, and numerous host species being raised together in common grazing pastures. Nuclear and mitochondrial DNA markers of nematodes have been utilized for species differentiation 
and genetic variability studies (Chaudhry et al. 2014; Kandil et al. 2017).

The mtDNA has exhibited as a feasible molecular marker for evolutionary and developmental investigations in animal populations and has informed to be a vital tool for studying population patterns, phylogeny, and ancestors for a wide assorted variety of animals (Cerutti et al. 2010). Generally, mtDNA has a higher rate of substitution than does nuclear DNA (Blouin 2002; Gharamah et al. 2012), making it conceivable to determine variations among closely related individuals. Hence, sequence variations in cytochrome oxidase subunit I (COI) gene are considered to be functional candidates for hereditary assorted diversity, disease transmission studies, and population structure analysis (Archie and Ezenwa 2011).

Recently, data on sympatric species dissemination, diversity, and structure of Haemonchus isolates from Egyptian ruminant animals are lacking. Thus, the present study will contribute helpful epidemiological information on population genetics and hereditary structure of this economically significant nematode in Egypt.

\section{Methods}

\section{Sample preparation}

Forty-eight adult male Haemonchus worms were collected from the abomasa of four major domestic ruminants (sheep, goats, camels, and cattle) (12 from each species regardless of prevalence rate) representing three major localities (Cairo, Giza, and Qalubia) in Egypt. The animals were showing clinical signs of possible haemonchosis including poor body condition, loss of hair or wool, emaciation, and anemic mucosa. These possibly infected ruminants were slaughtered in different abattoirs in Egypt. The worms were identified morphologically, according to Lichtenfels et al. (1994) and stored in 70\% ethanol.

\section{Genomic DNA extraction}

Total genomic DNA was extracted from individual male worms using QIAmp DNeasy blood and tissue kit (Qiagen, Germany) according to the manufacturers protocol (Hussain et al. 2014). The DNA extracts were stored at $-20{ }^{\circ} \mathrm{C}$ prior to use.

\section{DNA amplification by polymerase chain reaction (PCR)}

PCR was performed according to Kanzaki and Futai (2002) in order to amplify mitochondrial COI gene for genetic diversity analysis using appropriate primer sets: NEMAT-F $\left(5^{\prime}\right.$ CCTACTATAATTGGTGGGTTTGGTAA3') as a forward primer and NEMAT-R(5'TAGCCGCAGTAAAAT AAGC ACG $3^{\prime}$ ) as a reverse primer. The thermocycling condition for amplification of the target gene included denaturation at $95^{\circ} \mathrm{C}$ for $50 \mathrm{~s}$, Annealing at $55^{\circ} \mathrm{C}$ for $45 \mathrm{~s}$ and extension at $72{ }^{\circ} \mathrm{C}$ for $45 \mathrm{~s}$. PCR products of amplified COI target gene were subjected to agarose gel electrophoresis in order to demonstrate the PCR products according to Sambrook et al. (1989).

\section{Direct sequencing}

Purified PCR amplicon were sequenced by means of an automated sequencer (ABI-3730, XL) at the Animal Health Research Institute (AHRI, Egypt). The previously mentioned forward and reverse primers were used for sequencing of such amplicon.

\section{Data analysis}

The raw sequences were edited using MEGA 6.0 program (Tamura et al. 2007). Sequences of COI amplified fragments were aligned using nucleotide BLAST program of NCBI (http://www.ncbi.nlm.nih.gov/BLAST/2. Multiple alignments of all the sequences following Clustal W algorithm for each of the three fragments were performed using Codoncode Aligner. Intra-population diversity parameters including nucleotide diversity, haplotype diversity, and average number of nucleotide differences were calculated using DnaSP, version 4.10 (Rozas 2009). Pairwise FST among Haemonchus populations and analysis of molecular variance (ANOVA) was performed using ARLEQUIN version 3.1 program developed by Excoffier et al. (2005) to evaluate genetic variance between and within populations, and between groups. To investigate the population structure, pairwise FST values were utilized to perform multidimensional scaling (MDS) analysis using SPSS version 22.0 (Hussain et al. 2014).

\section{Results}

In this study, for $H$. contortus worms isolated from the abomasa of suspected infected ruminants, mtDNA COI genes were utilized to determine the presence and the extent of genetic variations of Haemonchus spp. populations among four major domestic ruminants (sheep, goats, camels, and cattle) in Egypt. Sequence alignment of each worm isolated from same animal species showed the same nucleotide sequence even from different localities in Egypt so each worm sequence was represented in one sequence of sheep, goats, camels, and cattle. The population structure was elucidated by comparing the sequences from Egypt with the sequences of Haemonchus isolates from other countries published in GenBank.

An approximately 709-bp length of a partial COI gene from different ruminants was successfully amplified, and a band specific to Haemonchus spp. was obtained in all reactions using COI-specific primers (Fig. 1).

The specificity of PCR products was proved by sequencing of DNA amplicon. The resulted nucleotide sequences were edited utilizing MEGA 6.0 program 


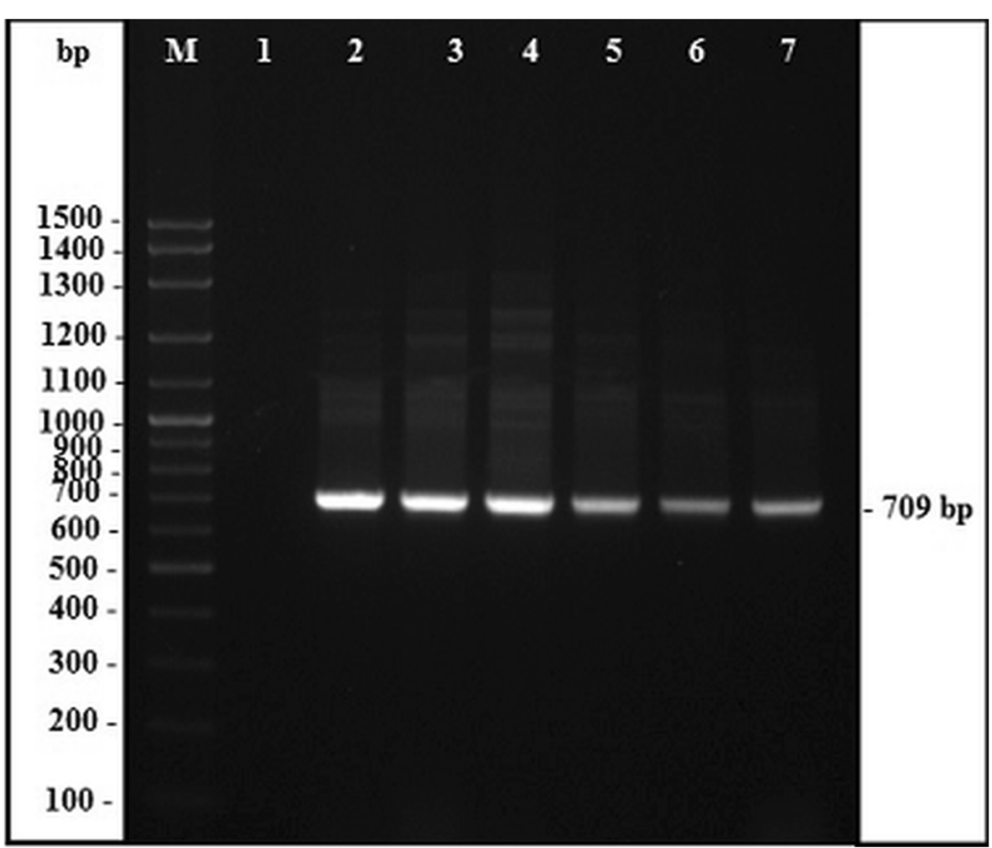

Fig. 1 Gel electrophoresis stained with ethidium bromide showing amplicons of COI gene detected at 709 bp. Lane M: DNA ladder 100 bp plus (Vivantis, Malaysia). Lane 1: Control negative. Lanes 2-4: Amplicon from sheep. Lane 5: Amplicon from goats. Lane 6: Amplicon from camels. Lane 7: Amplicon from cattle

producing a 262-bp length of target size that corresponded to nucleotide position 288 to 547 of the entire mitochondrial genome of Haemonchus worms and submitted in GenBank with accession numbers (KT826575, KT826574, KT826573, and KT826572) from sheep, goats, cattle, and camels, respectively. Analysis of the nucleotide sequence of PCR amplicons found that they belonged to the COI gene of $H$. contortus in sheep, goat, and cattle isolates but belonged to that of $H$. longistipes in the camel isolate.

The sequences of the local Egyptian Haemonchus spp. from four different ruminants were compared over an alignment length of $240 \mathrm{bp}$. The $\mathrm{G}+\mathrm{C}$ contents of COI sequences of sheep, goat, cattle, and camel isolates were $32 \%, 31.2 \%, 35.1 \%$, and $32.8 \%$, respectively. The COI fragments for four isolates studied identical in $76.3 \%$; the sequences coincided for 200 out of 262 nucleotides. Nine basic regions of interspecific homology (positions 27-34, 42-58, 84-98, 102-115, 132-139, 150-157, 183-191, 221-232, and 239) can be distinguished. Sequence difference between isolates occurred at 60 positions, comprising 56 substitutions (point mutations) and four deletions/insertions. Single base deletion was reported for positions 11 and 235, in sheep isolate, and only for position 11 in camel isolate.

The percent of identity and diversion between the Egyptian Haemonchus isolates from different hosts was reported. The highest identity percent was 93.5\% between sheep and goat isolate with divergence percent of $4.4 \%$. The lowest identity percent was $80.2 \%$ between sheep and camel isolates with divergence percent of $21.9 \%$. The identity percent of sheep isolate were 93.5 , 88.9 , and $80.2 \%$ with divergence percent of $4.4,9.6$ and $21.9 \%$ with goat, cattle, and camel isolates, respectively. The identity percent of goat isolate were 93.5 and $81.7 \%$ with divergence percent of 6.1 and $19.8 \%$ with cattle and camel isolates, respectively. The identity percent of cattle isolate was $83.6 \%$ with divergence of $17.3 \%$ with camel isolate.

The phylogenetic analysis in Fig. 2 showed a phenogram depicting genetic similarity of Haemonchus COI sequences based from pairwise sequence comparisons. $H$. longistipes isolated from camels was the genetically most distinct taxa. The phylogenetic tree showed clustering of sheep and goat $H$. contortus isolates which differ well from cattle $H$. contortus isolates.

The sequences were first aligned using Clustal W (1.82) program, and the phylogenetic analyses were conducted using PHYLIP package. Nucleotide substitutions are shown underneath the tree.

In order to further comprehend the population structure, a correlation of the partial genomic sequences (240 bp) of Haemonchus COI gene resulted from different hosts in Egypt with sequences of ten reference genotype sequences retrieved from GenBank from other countries and genus (Fig. 3). Egyptian sequences from sheep, goats, and cattle showed little variations among all published sequences which ranged from seven to ten 


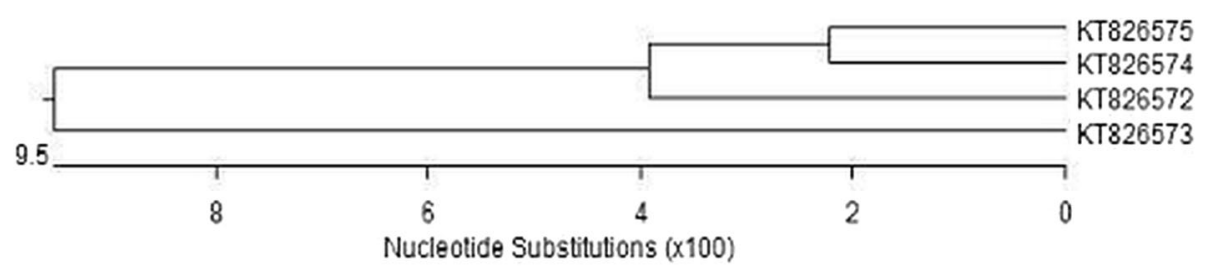

Fig. 2 Phylogenetic tree of the Egyptian Haemonchus isolates resolved from nucleotide sequences encoding for COI gene

substitutions (Fig. 3). On the other hand, Egyptian sequence from camels demonstrated great variation with others including 30 substitutions. The percent of identity and diversion between the Egyptian isolates and reference strains from the GenBank data was reported. Our isolates showed highest identities with Haemonchus isolated from Pakistan involving accession numbers KJ724402 (94.9\% identity and 4\% divergence with sheep isolate), KJ724377 (98.3\% identity and $1.7 \%$ divergence with goat isolate), and KJ 724399 (96.2\% identity and 2.6\% divergence with cattle isolate), while camels isolate had the highest identity with $H$. longistipes isolated from Pakistan (KJ724419 with $97.4 \%$ identity and $2.6 \%$ divergence).

The phylogenetic analysis of aligned COI sequences of these Egyptian isolates from different hosts and countries (Fig. 3) demonstrated discrete clusters and grouping that revealed close ancestral and relative genetic origin among those retrieved from GenBank. The Neighbor-Joining (NJ) dendrogram generated with 26 replicates (Fig. 3) revealed four main clades. The first clade consisted of $H$. contortus while the second clade included H. placei and Trichostrongylus axei. The other two clades consist of $H$. longistipes isolates and Cooperia oncosphora.

\section{Discussion}

Molecular technique recommend some advantages over morphology-based detection in that it is more objective, is more scalable, is easier to implement, and permits rapid characterization of Haemonchus spp. to address the shortcoming of the usual diagnosis of parasitic gastroenteritis.

The genome of Haemonchus spp. in Egypt was investigated at the molecular level as a preliminary step toward Haemonchus spp. evolutionary genetic structure through reception of genome sequencing strategies which will rapidly enhance the knowledge of the virulence and drug resistance of parasites. Only adult male worms were utilized for DNA amplification by PCR in order to avoid the danger of temperamental DNA enhancement from the eggs of female worms (Gharamah et al. 2012).

Mitochondrial DNA genome has a higher incidence of substitution than does nuclear DNA, making it conceivable to determine contrasts between closely related individuals (Blouin 2002), so it is considered to be a rich source of molecular markers for various scopes involving population genetics and taxonomic studies $(\mathrm{Hu}$ and Gasser 2006). Different approaches reported the genetic diversity in Haemonchus populations from various countries (Gharamah et al. 2012; Yin et al. 2013). There are two studies, in Brazil (Brasil et al. 2012) and in Pakistan (Hussain et al. 2014), in which the COI gene were applied. In our study, the predominance of $\mathrm{H}$. contortus in both sheep and goat hosts indicates that $H$. contortus is well-adapted and preferentially infects small

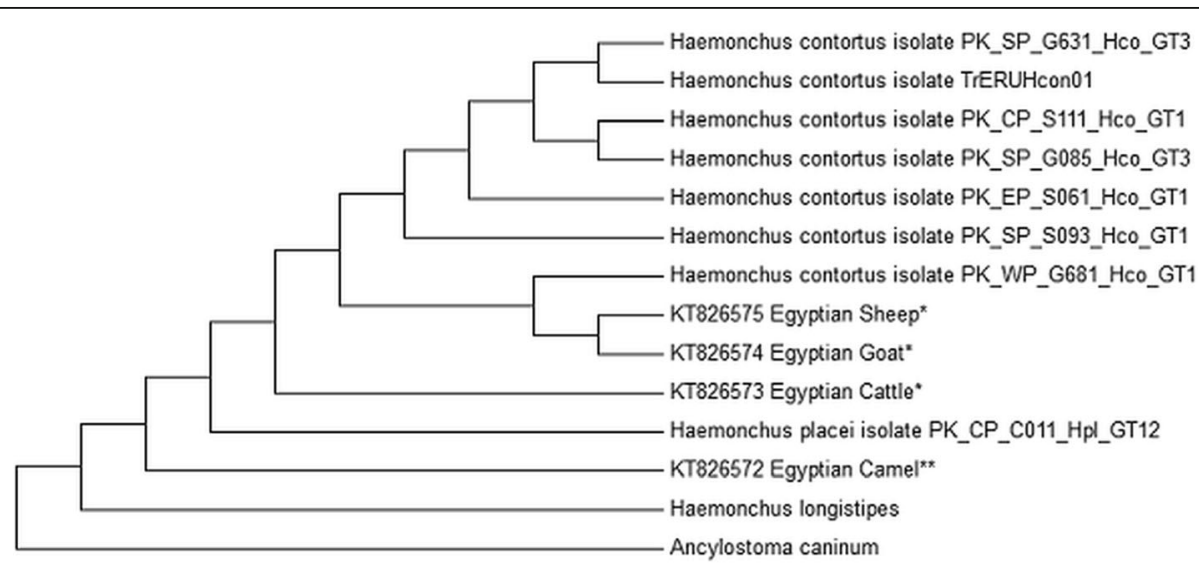

Fig. 3 Phylogenetic tree of Haemonchus COI gene isolates from Egypt and other countries with related species. Nucleotide substitutions are indicated below the tree 
ruminants, which are highly susceptible and the primary host of this species. This observation is in concurrence with previous investigations (Akkari et al. 2013), in spite of the fact that $H$. placei is accepted to be specially a parasite of cattle (Brasil et al. 2012). In contrast, the current study revealed the emergence of $H$. contortus as cattle species in agreement with the result of Akkari et al. (2013) in Tunisia. The relative power of various sympatric Haemonchus spp. among ruminant hosts seems to rely upon the kind of livestock management system winning in various regions. In cattle pastures with mono-grazing species, $H$. placei was found to prevail while in pastures with rotational or multi-grazing ruminant species, significant emergence of $H$. contortus infections in cattle do occur (Chaudhry et al. 2014). In small holder production system as Egyptian breeds where multiple grazing of sheep, goats, and cattle are predominant, the majority of $H$. contortus infection in cattle could have been originated from grazing areas contaminated by infected sheep and/or goat manure. This proposes the incidence of cross infection and the wide range of ruminant hosts for Haemonchus spp. of domestic ruminants in Egypt. This work is the first study which proved genetically that $H$. longistipes is $H$. species of Egyptian camels.

The results revealed a genetic diversity among populations of Haemonchus spp., including those from different hosts (sheep, goats, cattle, and camels) in Egypt. The COI sequences of different hosts revealed a high frequency of major difference included 60 positions such as insertions, deletion in 4 positions and substitution in 56 positions. This result is in agreement with many investigators who indicated the variation of COI sequences among Haemonchus spp. from different hosts in the world: Brasil et al. (2012) in Brazil and Hussain et al. (2014) in Pakistan. It was noticed there is some homology between sheep and goat sequences which is closely related to cattle sequence so the phylogenetic investigation of COI sequences did not expose clustering of haplotypes generating from a specific host demonstrating a high rate of gene flow among Haemonchus parasites infecting sheep, goats, and cattle in Egypt. The obtained results agreed with those obtained by Brasil et al. (2012) who proved that high rates of gene flow exist among populations of Haemonchus spp. in Brazil and additionally among those from different ruminant hosts and Hussain et al. (2014) who observed high genetic variability in Pakistani Haemonchus isolates at COI gene loci with high rates of gene flow among Haemonchus parasites infecting domestic ruminants. In contrast, we found that $H$. longistipes isolated from Egyptian camels showed little homology with $H$. contortus isolated from sheep, goats, and cattle and was the genetically most distinct taxa without clustering with other hosts in phylogenetic analysis.
The high level of hereditary assorted variety noticed in Egyptian sequences is typical of trichostrongylids (Silvestre et al. 2009) and is believed to be an outcome of both parasite-related and host-related factors. Parasite components including a significant biotic potential, a large population size, rapid direct life cycle, and infection rate combined with the incredible mutation rates found in these extremely polymorphic nematodes or as a result of persistence of infective stage of Haemonchus within the environmental conditions which encourage cross-species migration (Hussain et al. 2014; Brasil et al. 2012) are additionally in concurrence with Riggs (2001) who discussed the distinctions in diversity parameters between Haemonchus spp. to variations in their productivity, prepatent period, host superiority, and evolutionary rate. The host components are as follows: (i) they originate from different ruminant species, (ii) the presence of hybrid infection and movements and the abundance of $H$. contortus among heterologous hosts, such as sheep, goats, and cattle sharing the same grazing areas, enhancing the transmission of infection from one host species then onto the next, (iii) the lack of anthelmintic selection and consequent effective population in intensively reared herds, and (iv) extensive gene flow crosswise over subpopulations resulting from the movements of hosts (Akkari et al. 2013). On the other hand, $H$. longistipes isolated from camels in Egypt did not show gene flow with $H$. contortus infecting Egyptian domestic ruminants.

In the present research, the isolates showed highest identity with Haemonchus isolated from Pakistan (KJ724402 with sheep isolate, KJ724377 with goat isolate, KJ 724399 with cattle isolate, and KJ724419 with camel isolate). We also noticed that $H$. contortus from sheep, goats, and cattle exit in the same Pakistani $H$. contortus cluster in the phylogenetic tree and in the same way as $H$. longistipes. There are two conceivable theories that could underpin the resulted pattern of clustering. First, is the circulation of host across the geographic region possible (West Asia and Africa) as discussed by Akkari et al. (2013)? Second, is the evolutionary divergence suggesting that both species are related to a same progenitor during their developmental history possible as discussed by Brasil et al. (2012)?

\section{Conclusion}

Various data were collected about DNA structure of Haemonchus nematodes from Egyptian sheep, goats, cattle, and camels. PCR and sequencing contribute precise strategies for the detection of true taxonomic classification of various genotypes. Sequence data facilitates a superior comprehension of the evolution and transmission of Haemonchus species at the farm level. Furthermore, genotyping of Haemonchus spp. isolated from Egyptian farm animals will guide the application of efficient control strategies. 


\section{Acknowledgements}

Not applicable.

\section{Funding}

The authors thankfully acknowledge the financial assistance of NRC and STDF which support our study through project "Genetic characterization and diversity patterns of Haemonchus species populations isolated from small ruminants in Egypt".

\section{Availability of data and materials}

The datasets used and/or analyzed during the current study are available from the corresponding author on reasonable request.

\section{Authors' contributions}

All authors equally worked, written, read, and approved the final manuscript.

\section{Ethics approval and consent to participate}

Not applicable.

\section{Consent for publication}

Not applicable.

\section{Competing interests}

The authors declare that they have no competing interests.

\section{Publisher's Note}

Springer Nature remains neutral with regard to jurisdictional claims in published maps and institutional affiliations.

\section{Author details}

'Department of Parasitology and Animal Diseases, National Research Centre (NRC), PO Box 12622, 33 Bohouth St., Dokki, Giza, Egypt. ²Department of Parasitology, Faculty of Veterinary Medicine, Benha University, PO Box 13736, Moshtohor, Toukh, Egypt.

\section{Received: 30 August 2018 Accepted: 6 November 2018}

\section{Published online: 27 November 2018}

\section{References}

Akkari H, Jebali J, Gharbi M, Mhadhbi M, Awadi S, Darghouth MA (2013) Epidemiological study of sympatric Haemonchus species and genetic characterization of Haemonchus contortus in domestic ruminants in Tunisia. Vet Parasitol 193:118-125

Archie EA, Ezenwa VO (2011) Population genetic structure and history of a generalist parasite infecting multiple sympatric host species. Int J Parasitol 41:89-98

Blouin MS (2002) Molecular prospecting for cryptic species of nematodes: mitochondrial DNA versus internal transcribed spacer. Int J Parasitol 32:527-531

Brasil BS, Nunes RL, Bastianetto E, Drummond MG, Carvalho DC, Leite RC, Molento MB, Oliveira DA (2012) Genetic diversity patterns of Haemonchus placei and Haemonchus contortus populations isolated from domestic ruminants in Brazil. Int J Parasitol 42:469-479

Cerutti MC, Citterio CV, Bazzocchi C, Epis S, D'Amelio S, Ferrari N, Lanfranchi P (2010) Genetic variability of Haemonchus contortus (Nematoda: Trichostrongyloidea) in alpine ruminant host species. J Helminthol. 83:276-283

Chaudhry U, Miller M, Yazwinski T, Kaplan R, Gilleard J (2014) The presence of benzimidazole resistance mutations in Haemonchus placei from US cattle. Vet Parasitol 204:411-415

Elshahawy IS, Metwally AM, Ibrahim DA (2014) An abattoir-based study on helminthes of slaughtered goats (Capra hircus L., 1758) in upper Egypt, Egypt. Helminthologia 51:67-72

Excoffier L, Laval G, Schneider S (2005) Arlequin ver.3.0: an integrated software package for population genetics data analysis. Evol Bioinforma 1:47-50

Gasser RB, Bott NJ, Chilton NB, Hunt P, Beveridge I (2008) Toward practical, DNAbased diagnostic methods for parasitic nematodes of livestock-bionomic and biotechnological implications. Biotechnol Adv 26:325-334

Gharamah AA, Siti Azizah MN, Rahman WA (2012) Genetic variation of Haemonchus contortus (Trichostrongylidae) in sheep and goats from Malaysia and Yemen. Vet Parasitol 188:268-276
Hu M, Gasser RB (2006) Mitochondrial genomes of parasitic nematodes progress and perspectives. Trends Parasitol 22:78-84

Hussain T, Periasamy K, Nadeem A, Babar ME, Pichler R, Diallo A (2014) Sympatric species distribution, genetic diversity and population structure of Haemonchus isolates from domestic ruminants in Pakistan. Vet Parasitol 206: 188-199

Kandil OM, Abdelrahman KA, Fahmy HA, Mahmoud MS, El Namaky AH, Miller JE (2017) Phylogenetic patterns of Haemonchus contortus and related trichostrongylid nematodes isolated from Egyptian sheep. J Helminthol 91:583-588

Kandil OM, Eid NA, Elakabaw LM, Abdelrahman KA, Helal MA (2015) Immunodiagnostic potency of different Haemonchus contortus antigens for diagnosis of experimentally and naturally Haemonchosis in Egyptian sheep. Acta Parasitologica Globalis 6:246-255

Kanzaki N, Futai K (2002) A PCR primer set for determination of phylogenetic relationships of Bursaphelenchus species within the xylophilus group. Nemat 4:35-41

Lichtenfels JR, Pilitt PA, Hoberg EP (1994) New morphological characters for identifying individual specimens of Haemonchus spp. (Nematoda: Trichostrongyloidea) and a key to species in ruminants of North America. J Parasitol 80:107-119

Riggs NL (2001) Experimental cross-infections of Haemonchus placei (place, 1983) in sheep and cattle. Vet Parasitol 94:191-197

Rozas J (2009) DNA sequence polymorphism analysis using DSP. Methods Mol Biol 537:337-350

Sambrook J, Fritsch EF, Maniatis T (1989) Molecular cloning: a laboratory manual. Cold Spring Harbor Laboratory Press, New York

Schafer AS, Leal MLR, Molento MB, Aires AR, Duarte MMMF, Carvalho FB, Tonin AA, Schmidt L, Flores EMM, Franc RT, Grando TH, Minho AP, Krause A, Antoniazzi AQ, Lopes STA (2015) Immune response of lambs experimentally infected with Haemonchus contortus and parenterally treated with a combination of zinc and copper. Small Rumin Res 123:183-188

Silvestre A, Sauve C, Cortet J, Cabaret J (2009) Contrasting genetic structures of two parasitic nematodes determined on the basis of neutral microsatellite markers and selected anthelmintics resistance markers. Mol Ecol 18:5086-5100

Tamura K, Dudley J, Nei M, Kumar S (2007) MEGA 4: molecular evolutionary genetics analysis (MEGA) software version 4.0. Mol Bio and Evol 24:1596-1599

Taylor MA (2010) Parasitological examinations in sheep health management. Small Rumin Res 92:120-125

Whitley NC, Oh SH, Lee SJ, Schoenian S, Kaplan RM, Storey B, Terrill TH, Mobini S, Burke JM, Miller JE, Perdue MA (2014) Impact of integrated gastrointestinal nematode management training for U.S. goat and sheep producers. Vet Parasitol 200:271-275

Yin F, Gasser RB, Li F, Bao M, Huang W, Zou F, Zhao G, Wang C, Yang X, Zhou Y, Zhao J, Fang R, Hu M (2013) genetic variability within and among Haemonchus contortus isolates from goats and sheep in China. Parasit Vectors 6:279-287

\section{Submit your manuscript to a SpringerOpen ${ }^{\circ}$ journal and benefit from:}

- Convenient online submission

- Rigorous peer review

- Open access: articles freely available online

High visibility within the field

- Retaining the copyright to your article

Submit your next manuscript at $\boldsymbol{\nabla}$ springeropen.com 